\title{
Control del balance energético negativo y comportamiento productivo y metabólico en vacas doble propósito bajo suplementación energética
}

\author{
Control of negative energy balance \\ and productive and metabolic behavior in dual \\ purpose cows under energy supplementation
}

\section{Controle do balanço energético negativo e comportamento produtivo e metabólico em vacas duplo propósito baixo suplementação energética}

Laura Gómez Ortiz¹ \& Rómulo Campos Gaona²

1Zootecnista. Magister en Ciencias Agrarias. Estudiante de Doctorado en Ciencias Agrarias en la Universidad Nacional de Colombia, Sede Palmira. Colombia. ${ }^{2}$ Médico Veterinario. Magister en Medicina Veterinaria.

1,2Laboratorio de Nutrición Animal. Facultad de Ciencias Agropecuarias. Universidad Nacional de Colombia. Sede Palmira. Carrera 32 No 12 - 00 Chapinero, Vía Candelaria Palmira - Valle del Cauca - Colombia. Doctor en Ciencias Veterinarias

11lgomezo@unal.edu.co,

${ }^{2}$ rcamposg@unal.edu.co

\section{Resumen}

El objetivo de esta investigación fue evaluar el efecto de un suplemento energético de alta densidad sobre el control del balance energético negativo y el comportamiento productivo y metabólico durante el período de transición de vacas doble propósito, para este fin se utilizaron 14 vacas de dos a tres partos, con producciones promedio de 15 I/día y que estuvieran en los 15 días próximos al parto, las vacas se distribuyeron en dos grupos aleatoriamente. Las vacas del tratamiento $1(n=7)$ recibieron $150 \mathrm{~g}$ de propilenglicol $(\mathrm{PG})$ vía oral cada tercer día durante 90 días y el tratamiento control $(n=7)$ no recibió suplementación. Se obtuvieron 8 muestras de sangre a partir del primer día y con intervalos de 15 días, hasta el día 75 después del parto. Se determinó la concentración sérica de NEFA, BHB y Glucosa, además se evaluó la producción de leche, la actividad reproductiva y la relación costo-beneficio de la suplementación. Los resultados fueron analizados bajo un diseño mixto de medidas repetidas en el tiempo utilizando el software InfoStat (2008). Se encontraron diferencias significativas para todos los metabolitos evaluados. La producción de leche aumentó en un $13.4 \%$ para los animales suplementados con PG y se mejoró la actividad reproductiva. Los resultados permiten concluir que la suplementación con propilenglicol tiene un efecto positivo sobre el control del balance energético negativo en vacas doble propósito.

Palabras clave: suplementación, balance energético negativo, periodo de transición, propilenglicol. 


\section{Abstract}

The objective of this research was to evaluate the effect of an energy supplement of high density of negative energy balance control and behavior productive and metabolic during the period of transition from cows dual purpose, for this purpose 14 cows from two to three deliveries, with average yields of $15 \mathrm{l} /$ day were used and were in the next birth 15 days, the cows were distributed into two groups randomly. The cows of the treatment 1 (n = 7) received $150 \mathrm{~g}$ of propylene glycol $(\mathrm{PG})$ via oral every third day for 90 days and the treatment control $(n=7)$ not received supplementation. It obtained 8 samples of blood starting from the first day and with intervals of 15 days, until the day 75 after the childbirth. Serum concentration of NEFA, BHB and glucose was determined, also assessed milk production, reproductive activity and the relationship cost-benefit from supplementation. The results were analyzed under a joint design of repeated measurements in time using the InfoStat (2008) software. They found differences significant for all those metabolites evaluated. The production of milk increased in a $13.4 \%$ for the animals supplemented with $P G$ and is improved the activity reproductive. The results suggest that supplementation with propylene glycol has a positive effect on the control of negative energy balance in dual purpose cows.

Key-words: supplementation, negative energy balance, transition period, propyleneglicol.

\section{Resumo}

O objetivo desta pesquisa foi avaliar o efeito de um suplemento energético de alta densidade sobre o controle do balanço energético negativo e o comportamento produtivo e metabólico durante o período de transição de vacas de duplo propósito. Com essa finalidade foram usadas 14 vacas de dois e três partos com produções em média de 15 1/dia e que estivessem nos 15 dias próximos ao parto. As vacas foram distribuídas ao acaso. As vacas do tratamento1( $n=7)$ receberam $150 \mathrm{~g}$ de propilenglicol $(\mathrm{PG})$ via oral cada terceiro dia ao longo de 90 dias. O tratamento controle $(n=7)$ não recebeu suplemento. Obtiveram-se 8 amostras de sangue desde o primeiro dia e com intervalos de 15 dias, até o dia 75 depois do parto. Foi determinada a concentração sérica de NEFA, BHB e Glucose, além disso foi avaliada a produção de leite, a atividade reprodutora e a relação custo-benefício da suplementação. Os resultados foram analisados baixo um desenho experimental misturado de medições repetidas no tempo utilizando-se o programa InfoStat (2008). Foram encontradas diferenças significativas para todos os metabolitos avaliados. A produção de leite incrementou em $13,4 \%$ para animais com suplemento PG e melhorou a atividade reprodutora. Esses resultados permitem concluir que a suplementação com propilenglicol tem efeito positivo no controle do balanço energético negativo nas vacas de duplo propósito.

Palavras-chave: suplementação, balanço energético negativo, período de transição, propilenglicol.

\section{Introducción}

El balance energético negativo (BEN) es universal en las vacas lecheras durante las primeras semanas de lactancia, resultando que la mayoría de ellas lo soportan sin desarrollar enfermedades peripartales durante el intento de adaptar su metabolismo al BEN (Cardoso, 2008) sin embargo, las vacas lecheras (Bos taurus) de alta producción, desde el periodo preparto hasta la octava semana posparto presentan balance energético negativo (BEN) no compensado debido a la disminución del consumo de materia seca en el preparto y al incremento en la demanda energética para la producción de leche en el posparto, que conlleva a una movilización lipídica para suplir sus requerimientos energéticos (Cardoso, 2008).

Durante la vida de la vaca lechera es necesario que pase por diferentes procesos, nacimiento, destete y parto (Stalling, 1999), pero de todos estos procesos, es el periodo de transición asociado al parto, 
el más importante por todas las implicaciones que tiene sobre la presentación de diversas disfunciones metabólicas, productivas, reproductivas y sanitarias que pueden poner en riesgo la vida misma del animal (Correa, 2004). En el posparto y el inicio de la lactancia, se presenta un acelerado incremento en los requerimientos nutricionales (Correa, 2001), causando un balance energético negativo, que puede prolongarse durante varias semanas. En condiciones del trópico, la nutrición de los animales está basada en pasturas de baja calidad nutricional que limitan el consumo de materia seca, al igual que la presión por estrés calórico, sumado a esto, las condiciones de manejo y la gran variabilidad genética, provocan que la condición corporal de las vacas sea de por sí baja, inclusive antes del parto (Dominguez, Garmendia, \& Martinez, 2007); como resultado, los animales en periodo de transición presentan procesos de movilización de tejidos grasos para cubrir sus requerimientos energéticos, esta movilización presenta diferentes grados de intensidad y afecta la homeostasis del animal.

Los precursores de energía tales como propilenglicol, suministrados en soluciones orales durante el periodo de transición, reducen la concentración de ácidos grasos no esterificados y cuerpos cetónicos, y aumentan la insulina y la glucosa en el plasma sanguíneo (Bell, 1995), lo que le permitirá al animal mostrar el comportamiento productivo y reproductivo de acuerdo a su potencial genético sin ser afectado por el desbalance nutricional. El estudio de respuestas fisiológicas a dicha suplementación, no sólo muestra diferentes alternativas en la nutrición del ganado lechero, sino que, puede ayudar a realizar un adecuado balance energético de la dieta del hato (Contreras, 2011).

\section{Materiales y métodos}

La investigación se llevó a cabo en un sistema doble propósito semi-intensivo localizado en el departamento del Valle del Cauca, Colombia, con una posición geográfica $3^{\circ}$ 37’ Norte, 76우 06' Oeste y $1706 \mathrm{msnm}$. Se utilizaron 14 animales con peso promedio de 450 a $630 \mathrm{~kg}$ que se encontraban entre segundo y tercer parto, de componente genético multirracial, con cruzamientos orientados hacia la producción de leche, con cruces 1/2, 3/8, 5/8, Bos Indicus x Bos taurus y creciente evolución hacia Bos Indicus. Los animales fueron seleccionados por encontrarse 15 días antes del parto y distribuidos aleatoriamente en dos grupos, el grupo control con 7 animales y el grupo suplementado con 7 animales, cada uno, al cual se le asignó un nivel de suplementación, con $150 \mathrm{~g}$ de propilenglicol de uso alimenticio denominado Tratamiento 1.

Los animales se encontraban bajo un sistema de pastoreo rotacional con pasto estrella (Cynodon plectostachium) suplementado con concentrado comercial según merito productivo (relación media 2:1) (Tabla 1). La dieta se calculó mediante el software Spartan (Spartan Dairy Ration, Evaluator/Balancer, Versión 3.0). El propilenglicol fue suministrado después del ordeño de la mañana, la suplementación se inició 15 días antes del parto, con el fin de que las vacas tuvieran un período de acostumbramiento, y hasta los 75 días después del parto.

Tabla 1. Análisis Bromatológico de Pasto Estrella (Cynodon Plectostachium) y Concentrado Comercial

\begin{tabular}{l|c|c}
\hline & \multicolumn{2}{|c}{ Composición química } \\
\hline \multirow{2}{*}{ Nutriente } & $\begin{array}{c}\text { Cynodon } \\
\text { plestoctachium }\end{array}$ & $\begin{array}{c}\text { Concentrado } \\
\text { comercial }\end{array}$ \\
\cline { 2 - 3 } & Valor en \% & Valor en \% \\
\hline Materia Seca & 22,97 & 92.61 \\
\hline Proteína & 17.07 & 18.71 \\
\hline FDN & 68.91 & 28.89 \\
\hline FDA & 38.65 & 11.81 \\
\hline Carbohidrato & 1.69 & 38.22 \\
\hline Celulosa & 31.54 & 8.64 \\
\hline Hemicelulos & 30.28 & 17.08 \\
\hline Lignina & 7.12 & 3.20 \\
\hline EE & 2.64 & 6.91 \\
\hline Cenizas & 9,69 & 7.27 \\
\hline
\end{tabular}


La muestra fue realizada con 2 repeticiones y los datos expresados en base seca. El factor de conversión de nitrógeno a proteína es de 6.25. Fuente: Laboratorio Nutrición Animal Universidad Nacional de Colombia Sede Palmira.

Las variables a analizar fueron: producción de leche, actividad reproductiva, relación costo beneficio, NEFA (sigla proveniente del inglés Non Esterified Fatty Acids), Betahidroxibutirato, y glucosa.

Las muestras de sangre se realizaron entre 6 y 7 am por venopunción coccígea y se centrifugaron a 2700 rpm durante 15 minutos y se almacenaron en alícuotas a $-20^{\circ} \mathrm{C}$, hasta que fueron recolectadas todas las muestras del experimento, luego fueron analizadas mediante pruebas enzimáticas colorimétricas para reflectometría óptica con kits comerciales Randox (Antrim, UK); para equipo de reflectometria óptica automatizada RT-1904C (Rayto, Shenzen, China).

Los datos recolectados fueron analizados bajo un diseño mixto de medidas repetidas en el tiempo utilizando el software InfoStat (2008). El número de lactancias, el tiempo y su interacción constituyeron los efectos fijos y la vaca, anidada en el periodo de muestreo, representó el efecto aleatorio. Tres estructuras de covarianza se evaluaron en su capacidad de ajuste, componente simétrico (CS), no estructurada (UN) y autorregresiva de primer orden (AR (1)). El menor valor del criterio de información de Akaike (AIC) y del criterio de información bayesiano (BIC) fueron utilizados como métodos para determinar la mejor estructura de covarianza (Littell et.al, 1998 citado por Posada et al., 2012). La estimación y comparación de las medias se realizó usando la instrucción ESTIMATE. Un análisis descriptivo exploratorio de tipo unidimensional, incluyendo media y desviación estándar, también fue realizado.

\section{Resultados y Discusión}

\section{NEFA (Non Esterified Fatty Acids - Ácidos Gra- sos no esterificados)}

Para NEFA se obtuvieron resultados comparables con los obtenidos por otros autores, se encontraron diferencias significativas entre el control y el tratamiento PG $(p<0.05)$ como se muestra en la Tabla 2 con los resultados de las medias obtenidas en el análisis estadístico.

Tabla 2. Medias Estadísticas de los Tratamientos para Niveles Séricos de Nefa.

\begin{tabular}{l|c|c|c}
\hline TRATAMIENTO & MEDIAS & E.E & $\mathbf{p}<0.05$ \\
\hline CONTROL & 0,41 & 0,04 & $\mathrm{~A}^{*}$ \\
\hline PROPILENGLICOL & 0,37 & 0,03 & $\mathrm{~B}^{*}$ \\
\hline
\end{tabular}

*Medias con una letra común no son significativamente diferentes $(p>0.05)$.

Múltiples estudios han informado que las concentraciones séricas de NEFA durante el preparto deben estar entre $0.3 \mathrm{mmol} / \mathrm{L}$ a $0.5 \mathrm{mmol} / \mathrm{L}$ (LeBlanc, Leslie \& Duffield, 2005; Ospina Nydam, Stokol \& Overton, 2010; Chapinal, 2011; Roberts et al., 2012) y durante el pos-parto entre 0,70 a $1,0 \mathrm{mmol} / \mathrm{L}$ (LeBlanc et al., 2005; Ospina et al., 2010; Chapinal et al., 2011; Roberts et al., 2012), lo cual coincide con los valores séricos de NEFA encontrados en esta investigación.

Para cada tratamiento hubo diferencias significativas $(p<0.05)$ entre los días evaluados, para el control hubo diferencias significativas entre el día - 15 y los otros días de muestreo, para el grupo suplementado con PG hubo diferencias significativas $(p<0.05)$ entre los días $-15,60$ y los otros días evaluados como lo muestra la Figura 1.

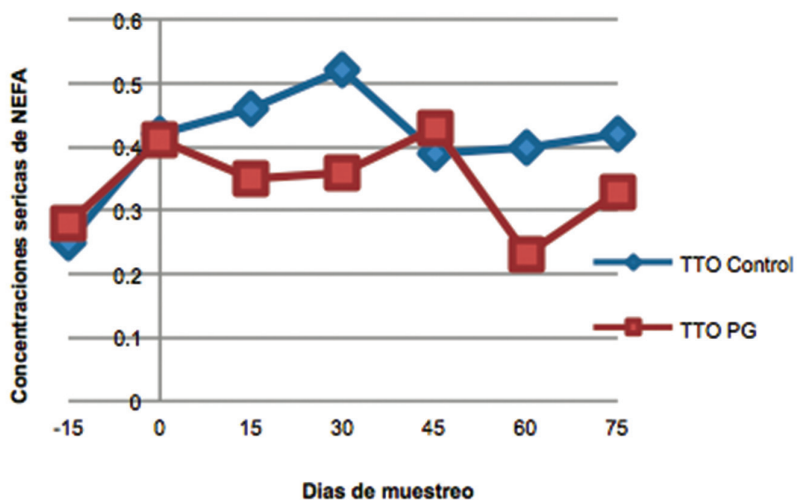

Figura 1. Concentraciones séricas de NEFA en vacas lecheras sometidas a dos tratamientos durante el periodo de transición. 
Los niveles séricos de NEFA aumentaron durante la semana del parto para el tratamiento PG, durante las dos semanas posteriores al parto los niveles de NEFA disminuyeron para el tratamiento $P G$, alcanzando un valor de $0.35 \pm 0.07 \mathrm{mmol} / \mathrm{L}$, estos valores sugieren que a pesar de que se puede reducir la respuesta insulínica mediada por un efecto lipogénico, los niveles séricos de NEFA pueden reducirse a través de la inhibición de la movilización grasa producida por el propilenglicol (Stephenson et al., 1997).

El propilenglicol (propano-1,2-diol) (PG), es un compuesto que realiza un metabolismo similar al propionato por lo tanto, puede ser utilizado como un precursor gluconeogénico exógeno (Nielsen \& Ingvartsen, 2004), ya que controla la movilización de grasa a partir de la estimulación de la liberación de insulina, que tiene un efecto inhibidor de la movilización de grasa del tejido adiposo. Este efecto se manifiesta en la reducción de los niveles de NEFA en sangre y la reducción de los niveles de triglicéridos en el hígado (Studer, Grummer \& Bertics, 1993); bajo esta premisa, Hoedemaker et al. (2004) encontraron que vacas en periodo de transición alimentadas con PG redujeron significativamente las concentraciones séricas de NEFA, al igual que los resultados reportados por Rizos et al. (2008) que demostraron que con el suministro de PG mejoraron el balance energético negativo de los animales, resultante de una disminución del catabolismo de grasa corporal y los niveles séricos de NEFA.

\section{BHB (beta-hidroxi-butirato)}

Para BHB se encontraron diferencias significativas $(p>0.05)$ entre el control y el tratamiento PG como lo muestra la Tabla 3, demostrando así que el PG tiene un efecto reductor.

Tabla 3. Medias Estadísticas de los Tratamientos para Niveles Séricos de Bhb.

\begin{tabular}{l|c|c|c}
\hline TRATAMIENTO & MEDIAS & E.E & $\mathbf{p}<0.05$ \\
\hline CONTROL & 0,83 & 0,04 & $\mathrm{~A}^{*}$ \\
\hline PROPILENGLICOL & 0,65 & 0,04 & $\mathrm{~B}^{*}$ \\
\hline
\end{tabular}

*Medias con una letra común no son significativamente diferentes $(p>0.05)$.
Entre los días de muestreo para el tratamiento control, se encontraron diferencias significativas $(p<$ 0.05 ) entre los días $-15,0$ que corresponde al parto y los otros días muestreados que van del día 15 al 75 después del parto, para el tratamiento PG se encontraron diferencias significativas $(p<0.05)$ entre los días 45,60 y los otros periodos como lo muestra la Figura 2.

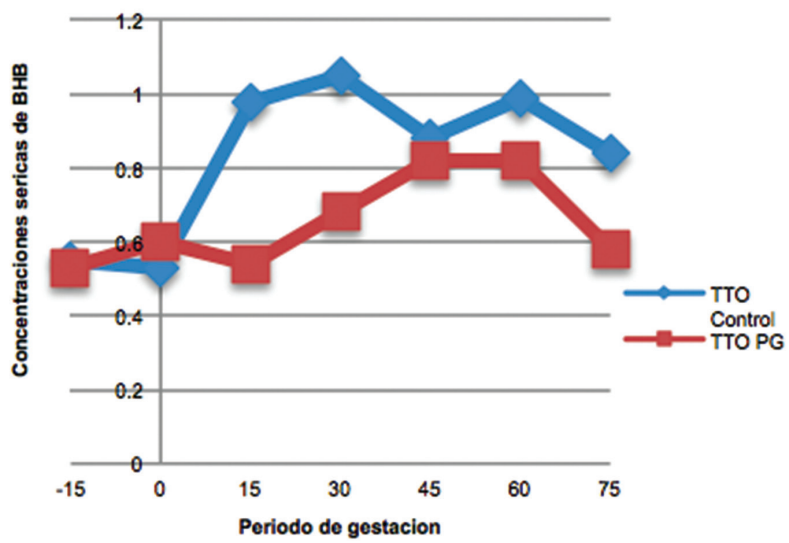

Figura 2. Concentraciones séricas de $B H B$ en vacas lecheras sometidas a dos tratamientos durante el periodo de transición.

Chapinal et al. (2011) indican que las concentraciones preparto por encima de $0.8 \mathrm{mmol} / \mathrm{L}$ se asocian con problemas post-parto, bajo las condiciones de esta investigación las concentraciones de BHB para el tratamiento $P G$ durante el periodo preparto ( -15 días con respecto al parto) tuvieron un valor medio de $0.65 \pm 0.02$ que se encuentra por debajo de los valores reportados en el anterior estudio.

Para el periodo pos-parto se reporta la presencia de enfermedades metabólicas con valores de BHB entre 1.2 y 1.4 mmol/L (Ospina et al., 2010; Chapinal et al., 2011; Seifi et al., 2007; Roberts et al., 2012), bajo las condiciones de esta invetigación no se encontraron valores medios para cada periodo por encima $0.90 \pm 0.06$, sin embargo, los animales del tratamiento control tuvieron siempre concentraciones séricas con valores superiores a las de PG lo que hace a estos animales más propensos a presentar enfermedades (Allen \& Piantoni, 2013). En estudios realizados por Rizos et al. (2008) se encontró que animales tratados con PG 
redujeron las concentraciones séricas de BHB, Hoedemaker et al. (2004) también reportaron que animales tratados durante el periodo de transición con PG tuvieron concentraciones séricas de BHB menores comparados con el tratamiento control, al igual que los resultados obtenidos en la presente investigación donde los valores de BHB para los animales tratados con PG fueron significativamente $(p<0.05)$ menores que los animales del grupo control, lo que sugiere que la disponibilidad de energía se mejoró con la suplementación de PG (Wang et al., 2009).

\section{Glucosa}

Según los valores de referencia de Kaneko et al. (2008) las concentraciones séricas normales de glucosa deben estar entre 2.5 y $4.16 \mathrm{mmol} / \mathrm{L}$ al igual que lo informado por Macrae et al. (2006) quienes consideran que los valores óptimos de glucosa deben ser $>3,0 \mathrm{mmol} / \mathrm{L}$, bajo las condiciones de este estudio las concentraciones séricas de glucosa tuvieron valores similares a los reportados por la literatura, sin embargo el tratamiento $P G$ difirió significativamente $(p<0.05)$ del tratamiento control según las medias estadísticas informadas en la Tabla 4

Tabla 4. Medias Estadísticas de los Tratamientos para Niveles Séricos de Glucosa

\begin{tabular}{l|l|l|l}
\hline Tratamiento & Medias & E.E & \multicolumn{1}{|c}{$\mathbf{p}<0.05$} \\
\hline Control & 3.05 & 0,09 & $\mathrm{~B}^{*}$ \\
\hline Propilenglicol & 3.39 & 0,09 & $\mathrm{~A}^{*}$ \\
\hline
\end{tabular}

*Medias con una letra común no son significativamente diferentes $(p>0.05)$

Para los días de muestreo se encontraron diferencias significativas para el tratamiento control entre el día 75 y los otros periodos, para el tratamiento PG no se encontraron diferencias significativas $(p>0.05)$ entre los periodos (Figura 3).

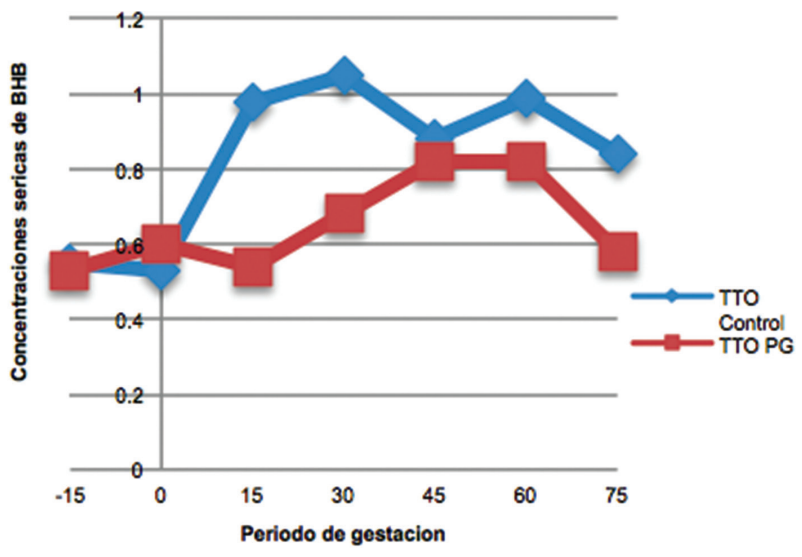

Figura 3. Concentraciones séricas de Glucosa en vacas lecheras sometidas a dos tratamientos durante el periodo de transición

Para los días de muestreo se encontraron diferencias significativas $(p<0.05)$ para los tratamientos en los días 30 y 45 que corresponden fisiológicamente al pico de la curva de lactancia, con respecto a los otros días de muestreados. Las concentraciones séricas de glucosa durante el preparto son bajas debido a la menor disponibilidad de sustratos para su formación como consecuencia de la disminución en el consumo de nutrientes y aumento de la demanda de nutrientes en el último tercio de la gestación (Bauman et al., 2000 citado por Strieder et al., 2014), estos niveles tienen un aumento durante el parto y luego caen nuevamente, este aumento se debe a un incremento en los glucocorticoides y glucagón, que agotan las reservas hepáticas de glucógeno, después de superadas las dos semanas pos-parto, los niveles séricos de glucosa se recuperan y aumentan parcialmente como consecuencia del incremento de la gluconeogénesis necesaria para la lactancia como se muestra en la Figura 3. (Doepel, Lobley, Bernier, Dubreuil \& Lapierre, 2009). Christensen Grummer, Rasmussen \& Bertic (1997), encontraron en su estudio resultados similares, donde la suplementación con PG fue más eficaz en el aumento de la glucosa sérica durante la lactancia que durante el preparto (Citado por Butler, Pelton \& Butl, 2006). 
Varios estudios describen un aumento significativo en los niveles séricos de glucosa en animales tratados con $\mathrm{PG}$ durante el periodo de transición (DeFrain, Hippen, Kalscheur \& Jardon, 2004; Wang et al., 2009), también se han encontrado mayores concentraciones de glucosa en vacas suplementadas con glicerol (Chung et al., 2007), siendo estos resultados consistentes con los encontrados bajo las condiciones de la presente investigación. El aumento en las concentraciones séricas de glucosa después del suministro de PG es debido a una disminución en la utilización de la glucosa por los tejidos periféricos, incluso bajo el aumento de los niveles séricos de insulina (Kristensen \& Raun, 2007). Esta resistencia insulínica en los tejidos periféricos fue probablemente inducida por el suministro oral de PG, una disminución en la proporción de metabolitos cetogénicos a glucogénicos en la sangre a partir del metabolismo $P G$, o ambos (Kristensen \& Raun, 2007).

\section{Producción de leche}

Para la producción de leche hubo diferencias significativas $(p<0.05)$ del tratamiento $P G$ con respecto al tratamiento control (Tabla 5) (Figura 4), estos resultados son comparables con los informados por Pickett et al. (2003) quienes encontraron que vacas suplementadas con propilenglicol durante el final de la gestación y el inicio de la lactancia incrementaron la producción de leche en un $12 \%$ con respecto al grupo control. El aumento de la producción de leche se puede esperar ya que el PG aumenta la concentración energética de la ración. PG es un aditivo con un alto contenido energético que se aproxima a 23.7 MJ de energía bruta/kg MS (Miyoshi, Pate \& Palmquist, 2001).
Tabla 5. Medias Estadísticas de los Tratamientos de Estudio para Producción de Leche

\begin{tabular}{l|l|l|l}
\hline TRATAMIENTO & Medias & E.E. & $\mathbf{p}<0.05$ \\
\hline CONTROL & 10,64 & 0,29 & $\mathrm{~B}^{*}$ \\
\hline PROPILENGLICOL & 11,43 & 0,26 & $\mathrm{~A}^{*}$ \\
\hline
\end{tabular}

*Medias con una letra común no son significativamente diferentes $(p>0.05)$.

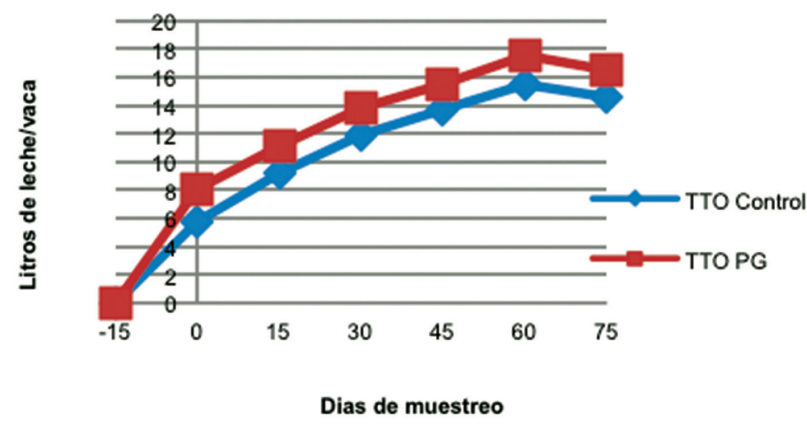

Figura 4. Leche producida por vacas sometidas a dos tratamientos durante el periodo de transición

\section{Relación costo-beneficio}

En Colombia un kg de propilenglicol se adquiere comercialmente por un valor de $\$ 7900$ pesos lo que representa un valor por g de $\$ 7.9$ pesos, cada animal fue suplementado para el tratamiento PG con $150 \mathrm{~g}$ durante 30 días. Para la suplementación oral del precursor energético se requieren 2 personas, en este caso personal del equipo de ordeño, los cuales reciben en Colombia un salario integral mensual por $\$ 800000$ pesos, este personal ocupa 10 minutos aproximadamente de su tiempo de trabajo suplementando cada animal lo que corresponde a $\$ 1092.89$ pesos por animal suplementado/día. En total el costo de la suplementación por animal durante todo el tratamiento se muestra en la Tabla 6.

Tabla 6. Costo Total de Suplementación por Animal Durante Todo el Tratamiento

\begin{tabular}{l|l|l|l|l|l}
\hline Suplemento & Costo PG/día & Días Tto & $\begin{array}{l}\text { Costo total } \\
\text { PG/30 días }\end{array}$ & $\begin{array}{r}\text { Costo de mano } \\
\text { de obra/ 30 días }\end{array}$ & Total \\
\hline$P G$ & $\$ 1.185,00$ & 30 & $\$ 35.550,00$ & $\$ 32.786,70$ & $\$ 68.336,70$ \\
\hline
\end{tabular}


Producir un litro de leche en Colombia cuesta $\$ 764$ pesos (País, 2013) para las condiciones de trópico bajo, suplementar una vaca con PG durante el periodo de transición bajo las condiciones de esta investigación tuvo un valor de \$22.14 pesos L/vaca/día lo cual corresponde a un costo total de producción de $\$ 786.14$ pesos L/vaca/día, este valor incrementa en un $2.8 \%$ los costos de producción para el hato, sin embargo, con respecto a la producción de leche del grupo control, los animales suplementados con propilenglicol obtuvieron en promedio $414 \mathrm{~L}$ más de leche durante el total de la lactancia lo que corresponde a un incremento de la producción de 13.4\%.

\section{Actividad reproductiva}

Durante el estudio se presentaron casos de repetición de celo después de la inseminación artificial para el tratamiento control que corresponde al $42 \%$ de los animales evaluados para el grupo, los animales suplementados con PG no presentaron esta condición reproductiva y quedaron preñadas dentro de los tiempos estimados en el hato, además se pasó de tener hasta 150 días abiertos a 110 días abiertos para los animales tratados con PG. Estudios realizados han demostrado que el balance energético negativo se asocia a una mayor incidencia de ciclos reproductivos irregulares que pueden aumentar al primer servicio y reducir las tasas de concepción (Wathes, Cheng \& Chowdhury, 2007). Este BEN ocasionado por el agotamiento prolongado de las reservas corporales y las fluctuaciones en la ingesta de materia seca durante el inicio de la lactancia, pueden tener efectos negativos importantes sobre la reactivación ovárica y las tasas de concepción (Ceballos, Villa, Betancourth, \& Roncancio, 2004). El mal estado energético del animal se ha asociado con menores tasas de recuperación de ovocitos y posteriores problemas en el desarrollo embrionario en las vacas lecheras (Butler, Pelton \& Butler, 2000).

Los efectos de reducción de niveles séricos de NEFA y BHB ocasionados por PG son un indicador de que la vaca está movilizando menos grasa y como consecuencia el balance de energía tiende a ser positivo (Nielsen \& Ingvartsent, 2004).
El balance negativo de energía excesivo y altos niveles de cuerpos cetónicos se han asociado con disminución de la eficiencia reproductiva en vacas lecheras (Trevisi, Amadori, Cogrossi, Razzuol, E, \& Bertoni, 2012) por lo tanto, la capacidad de PG para reducir la movilización grasa, especialmente en vacas con altos niveles de NEFA podría tener efectos beneficiosos en la reproducción.

\section{Conclusiones}

Haber suplementado con precursores energéticos, como el propilenglicol, PG, incrementó la producción de leche durante el periodo de transición.

La suplementación con PG corrigió parcialmente el balance energético negativo de las vacas y fue evidenciado en la reducción de los niveles séricos de NEFA y BHB y el aumento en los niveles séricos de glucosa.

La suplementación con PG mejoró la eficiencia reproductiva del hato con una menor incidencia en la repetición de celos después de la inseminación y la reducción de los días abiertos.

Suplementar una vaca con PG incremento en $13.4 \%$ la producción y sólo incrementó los costos de producción en un $2.8 \%$, por los tanto existe una relación positiva costo-beneficio.

\section{Literatuta Citada}

1. Allen, M. \& Piantoni, P. (2013). Metabolic Control of Feed Intake Implications for Metabolic Disease of Fresh Cows. Vet Clin Food Anim, 279-297.

2. Bauman, D. (2000). Regulation of nutrient partitioning during lactation: Homeostasis and homeorhesis. En Ruminant physiology: Digestion, Metabolism, Growth and Reproduction (págs. 311-28). Pretoria: Cronjé PB.

3. Bell, A. (1995). Regulation of organic nutrient metabolism during transition from late pregnancy to early lactation. . Journal of Animal Science, 73:2804-2819.

4. Butler, S., Pelton, S. \& Butler, W. (2006). Energy balance, metabolic status, and the first postpartum ovarian follicle wave in cows administered propylene glycol. $J$ Dairy Sci, 89:2938-51. 
5. Cardoso, F. (2008). Indicadores hematológicos, bioquímicos e ruminais no diagnóstico do deslocamento de abomaso à esquerda em vacas leiteiras do Sul do Brasil. Pseq agrop bras, 43:141-147.

6. Ceballos, A., Villa, N., Betancourth, T. \& Roncancio, D. (2004). Determinación de la concentración de calcio, fósforo y magnesio en el periparto de vacas lecheras en Manizales, Colombia. Rev Col Cienc Pec, Vol. 17:2.

7. Chapinal, N. C. (2011). The association of serum metabolites with clinical disease during the transition period. Journal of Dairy Science, 94, 4897-4903.

8. Christensen, J., Grummer, R., Rasmussen, F. \& Bertics, S. (1997). Effect of method of delivery of propylene glycol on plasma metabolites of feed-restricted cattle. J. Dairy Sci., 80, 563-568.

9. Chung, Y., Rico, D., Martínez, C., Cassidy, T., Noirot, V., Ames, A. et al. (2007). Effects of Feeding Dry Glycerin to Early Postpartum Holstein Dairy Cows on Lactational Performance and Metabolic Profiles. J. Dairy Sci. , 90:5682-5691.

10. Contreras, A. (2011). Lipid mobilization and inflammatory response during the transition period of dairy cows. Journal of Animal Science, 34:281-289.

11. Correa, H. (2001). Consumo de materia seca durante el periodo de transición. Curso de educación continuada., 3-15.

12. Correa, H. (2004). La vaca en transición: metabolismo y manejo nutricional. Seminario Nacional de lechería especializada: Bases Nutricionales y su impacto en la productividad. Eventos y Asesorias Agropecuarias (págs. 141-152). Medellin: Universidad Nacional de Colombia.

13. DeFrain, J. M., Hippen, A., Kalscheur, K. \& Jardon, W. (2004). Feeding glycerol to transition dairy cows: Effects on blood metabolites and lactation performance. J. Dairy Sci., 87:4195.

14. Doepel, L., Lobley, G., Bernier, J., Dubreuil, P. \& Lapierre, H. (2009). Differences in splanchnic metabolism between late gestation and early lactation dairy cows. J. Dairy Sci, 92 :3233-3243.

15. Dominguez, C., Garmendia, J. \& Martinez, N. (2007). Influencia de la época de parto, la condición corporal y la suplementación sobre la actividad ovárica postparto de vacas mestizas bajo pastoreo mixto en el norte del Estado Guárico. Revista de la Facultad de Ciencias Veterinarias, 48:37-50.

16. Hoedemaker, M., Prange, D., Zerbe, H., Frank, J., Daxenberger, A. \& Meyer, H. (2004). Peripartal propylene glycol supplementation and metabolism, animal health, fertility, and production in dairy cowa. J. Dairy Sci, 87:2136-2145.

17. Kaneko, J. (2008). Carbohydrate metabolims and its diseases. En J. Kaneko, J. Harvey, \& M. Bruss, Clinical Biochemistry of Domestic Animal (págs. 46-80). San Diego, California: Kaneko, J; Harvey, J; Bruss, M.

18. Kristensen, N. \& Raun, M. (2007). Ruminal and intermediary metabolism of propylene glycol in lactating Holstein cows. J. Dairy Sci., 90: 4707-4717.
19. LeBlanc, S., Leslie, K. \& Duffield, T. (2005). Metabolic predictors of displaced abomasum in dairy cattle. Journal of Dairy Science, 88, 159-170.

20. Littell, R. , Henry, P. \& Ammerman, C. 1998. Statistical analysis of repeated measures dara using SAS procedures. J. Animal Sci. 76:1216-1231

21. Macrae, A., Whitaker, D., Burrough, E., Dowell, A \& Kelly, J. (2006). Use of metabolic profiles for the assessment of dietary adequacy in UK dairy herds. Vet Rec., 155: 655-661.

22. Miyoshi, S., Pate, J. \& Palmquist, D. (2001). Propylene glycol for dairy cows $A$ review of the metabolism of propylene glycol and its effects on physiological parameters, feed intake, milk production and risk of ketosis. Anim. Reprod. Sci., 68:29-43.

23. Nielsen, N. \& Ingvartsen, K. (2004). Propylene glicol for dairy cows a review of the metabolism of propylene glycol and its effects on physiological parameter, feed intake, milk production and risk of ketosiscc. $J$ Dairy Sci, 115:191-213.

24. Ospina, P., Nydam, D., Stokol, T. \& Overton, T. (2010). Association between the proportion of sampled transition cows with increased nonesterified fatty acids and betahydroxybutyrate and disease incidence, pregnancy rate, and milk production at the herd level. Journal of Dairy Science, 93, 3595-3601.

25. País, R. (02 de Sep de 2013). 'Producir un litro de leche vale \$800'. EL TIEMPO.

26. Pickett, M. M., Piepenbrink, M. S. \& Overton, T. R. (2003). Effects of propylene glycol or fat drench on plasma metabolites, liver composition and production of dairy cows during the periparturient period. J. Dairy Sci. 86:2113-2121.

27. Posada, S., Noguera, R. \& Bedoya, O. (2012). Perfil metabólico de cabras lactantes de las razas Saanen y Alpina. Livestock Research for Rural Development, 1-9.

28. Rizos, D., Kenny, D., Griffin, W., Quinn, K., Duffy, P., Mulligan, F. et al. (2008). The effect of feeding propylene glycol to dairy cows during the early postpartum period on follicular dynamics and on metabolic parameters related to fertility. Theriogenology, 688-699.

29. Roberts, T., Chapinal, N., LeBlanc, S., Kelton, D., Dubuc, J. \& Duffield, T. (2012). Metabolic parameters in transition cows as indicators for early-lactation culling risk. J. Dairy Sci, 95:3057-3063.

30. Seifi, H., Gorji-Dooz, M., Mohri, M., Dalir-Naghadeh, B. \& Farzaneh, N. (2007). Variations of energy-related biochemical metabolites during transition period in dairy cows. Compendium of Clinical Pathology, 16, 253-258.

31. Stalling, C. (1999). Transition Cow Nutrition. Recuperado el 20 de Marzo de 2013, de Feed and Nutritional Management Cow College: www.dasc.vet. edu/nutrition.

32. Stephenson, K. A., Lean, I., Hyde, M., Curtis, M., Garvin, J. \& Lowe, L. (1997). Effects of monensin on the metabolism of periparturient dairy cows. J. Dairy Sci, 80:830-837. 
33. Strieder, C., Cucunubo, L., Smulders, J., Wittwer, F. \& Noro, M. (2014). Indicadores energéticos de vacas lecheras a pastoreo en período de transición y lactancia temprana con alta o moderada condición corporal preparto. Revista Científica, FCV-LUZ, Vol. XXIV, № 1, 73 - 82.

34. Studer, V., Grummer, R. \& Bertics, S. (1993). Effect of Prepartum Propylene Glycol Administration on Perlparturient Fatty Liver In Dairy Cows. J Dairy Sci , 76:2931-2939.

35. Trevisi, E., Amadori, M., Cogrossi, S., Razzuol, E. \& Bertoni, G. (2012). Metabolic stress and inflammatory response in high-yielding, periparturient dairy cows. Veterinary Science, 695-704.
36. Wang, C., Liu, Q., Yang, W., Huo, W., Dong, K., Huang, Y. et al. (2009). Effects of glycerol on lactation performance, energy balance and metabolites in early lactation Holstein dairy cows. Anim. Feed Sci. Technol, 151:12-20.

37. Wathes, D., Cheng, Z. \& Chowdhury, W. (2009). Negative energy balance alters global gene expression and immune responses in the uterus of postpartum dairy cows. 39 1-13. Physiological Genomics, 39:1-13. 